\title{
Vergleich von endobronchialem Ultraschall (EBUS) und Mediastinoskopie (MSK) beim Staging des Lungenkarzinoms
}

\section{Comparison of Endobronchial Ultrasound (EBUS) and Mediastinoscopy (MS) for Staging Lung Cancer}

Autoren

Institute
K. Kambartel ${ }^{1}$, T. Krbek ${ }^{2}$, Th. Voshaar ${ }^{1}$

${ }^{1}$ Lungenzentrum Bethanien Moers. Abteilung für Pneumologie, Allergologie, Zentrum für Schlaf- und Beatmungsmedizin (CA Dr. Th. Voshaar)

${ }^{2}$ Lungenzentrum Bethanien Moers. Abteilung für Thoraxchirurgie (CA Dr. T. Krbek) eingereicht $\quad$ 23.12. 2011 akzeptiert nach Revision 22. 3.2012

\section{Bibliografie}

Dol http://dx.doi.org/ 10.1055/s-0032-1309400 Online-Publikation: 24.4.2012 Pneumologie 2012; 66: 426-431 (c) Georg Thieme Verlag KG Stuttgart · New York ISSN 0934-8387

Korrespondenzadresse Dr. Kato Kambartel Bethanienstr. 21 47441 Moers kambartel@bethanienmoers.de

\section{Zusammenfassung \\ $\nabla$}

Ein mediastinaler Lymphknotenbefall ist von entscheidender Bedeutung für die Prognose und Therapie des nicht metastasierten Lungenkarzinoms. Der Stellenwert von endobronchialem Ultraschall mit transbronchialer Nadelaspiration (EBUS) und Mediastinoskopie (MSK) wird zwischen Pneumologen und Thoraxchirurgen oft kontrovers diskutiert. In dieser retrospektiven Studie wurde die diagnostische Genauigkeit des EBUS und der Mediastinoskopie bei Patienten mit einem gesicherten Lungenkarzinom untersucht. Zwischen Januar 2009 und Juli 2011 erfolgten bei 544 Lungenkrebspatienten 111 EBUS-Untersuchungen (z.T. in Kombination mit transoesophagealem Ultraschall mit Feinnadelaspiration (EUS) ) und 88 Mediastinoskopien. Die diagnostische Genauigkeit des EBUS (94\%) war der Mediastinoskopie (86\%) signifikant überlegen $(p<0,05)$. Der negative prädiktive Wert beim EBUS und MSK betrugen jeweils $83 \%$, die Sensitivität $94 \%$ bzw. 58\%, die Prävalenz eines N2/3-Stadiums 84\% bzw. $32 \%$ und die Komplikationsrate 0\% bzw. 3,4\%. Aufgrund der zumindest vergleichbaren diagnostischen Genauigkeit sollte die EBUS-TBNA (nach Möglichkeit in Kombination mit EUS) das erste Verfahren zur histologischen Beurteilung des Mediastinums bei Lungenkrebspatienten sein.

\section{Einleitung}

$\nabla$

Ein Tumorbefall der mediastinalen Lymphknoten ist ein entscheidender Faktor für die Prognose und Therapie des Lungenkarzinoms [1]. Durch bildgebende Verfahren wie CT oder PET kann oft keine ausreichend sichere Aussage über die Dignität der Lymphknoten getroffen werden [2]. Aus diesem Grund ist vor einer operativen Therapie in der Regel eine histologische Untersuchung des Mediastinums erforderlich. Nach den aktuellen Leitlinien ist die Mediastinoskopie hierzu der

\section{Abstract \\ $\nabla$}

Diagnostic findings of mediastinal metastasis are an important factor for the prognosis of and therapy for lung cancer. In this retrospective study we examined the role of endobronchial ultrasound with transbronchial needle aspiration (EBUS) and mediastinoscopy (MS) in patients with confirmed lung cancer. Between 01/2009 and 07/ 2011 we performed 111 EBUS procedures [partly in combination with transoesophageal endoscopic ultrasound-guided fine-needle aspiration (EUS-FNA)] and 88 mediastinoscopies. The diagnostic accuracy of EBUS (94\%) was superior to that of MS $(86 \%)(\mathrm{p}<0.05)$. The negative predictive value of EBUS and MS was $83 \%$ for both, the sensitivity was $94 \%$ vs. $58 \%$, the prevalence of N2/N3 was $84 \%$ vs. $32 \%$ and the rate of complications was $0 \%$ vs. $3 \%$. Due to the at least similar accuracy the EBUS should be the first diagnostic procedure for histological staging of the mediastinum in patients with lung cancer.
Goldstandard, da nur durch sie mit ausreichender Sicherheit ein mediastinaler LK-Befall ausgeschlossen werden kann $[3,4]$.

Der endobronchiale Ultraschall hat in den letzten Jahren eine zunehmende Bedeutung beim mediastinalen Staging erlangt [5-7]. Insbesondere durch die Kombination von EBUS und EUS kann fast das gesamte Mediastinum untersucht werden und es sind Lymphknotenstationen erreichbar, die durch eine zervikale Mediastinoskopie nicht erreicht werden können [8-10]. 
Tab. 1 Vergleich von EBUS und MSK zum pathologischen N-Stadium. Negative Befunde von EBUS und MSK sind durch eine Thorakotomie überprüft.

\begin{tabular}{|c|c|c|}
\hline \multicolumn{3}{|c|}{ Postoperativer Befund } \\
\hline Ergebnis & pNO/1 & $\mathrm{pN} 2 / 3$ \\
\hline \multicolumn{3}{|l|}{ EBUS } \\
\hline EBUS N 0/1 & 15 (korrekt) & 3 (falsch) \\
\hline EBUS N $2 / 3$ & 0 & 78 (korrekt) \\
\hline unklar & 1 & 2 \\
\hline \multicolumn{3}{|l|}{ MSK } \\
\hline MSK N0 / 1 & 50 (korrekt) & 10 (falsch) \\
\hline MSK N2 / 3 & 0 & 14 (korrekt) \\
\hline unklar & 0 & 1 \\
\hline
\end{tabular}

Der Stellenwert der EBUS-TBNA als Staginginstrument des Mediastinums im Routineeinsatz eines Lungenkrebszentrums soll anhand dieser retrospektiven Untersuchung analysiert werden.

\section{Material und Methoden \\ $\nabla$}

Im Lungenzentrum Moers werden seit 2009 alle Patienten mit einem Lungenkarzinom in einer Datenbank erfasst. Diese Datenbank ist in das Krankenhausinformationssystem integriert und ermöglicht so eine komplette Analyse diagnostischer Prozeduren.

Die Ergebnisse von EBUS-TBNA Untersuchungen und Mediastinoskopie wurden retrospektiv analysiert. Seit 2010 wurde in unserer Klinik bei Bedarf auch der endobronchiale Ultraschall mit dem transoesophagealen Ultraschall (EUS) kombiniert durchgeführt.

Alle EBUS (und EUS)-Untersuchungen erfolgten mit dem Olympus-EBUS-Punktionsbronchoskop BF-UC160F-OL8 im Rahmen einer starren Bronchoskopie in Allgemeinanästhesie. Die Lymphknotenstationen 2/4 R, 2/4L, 7, 10/11 R, 10/11 L und ggf. 5 wurden sonografisch beurteilt. Es erfolgte eine dreimalige Punktion (bei gutem Material zweimalige Punktion) der klinisch relevanten Lymphknoten. Die pathologische Aufarbeitung erfolgte je nach Material als Histologie oder Zytologie. Eine immunhistochemische Diagnostik war obligat.

Die Zuordnung der Lymphknotenstationen erfolgte 2009 nach Mountain und Dressler [11], ab 2010 nach IASLC-Map [12].

Die EBUS-Untersuchungen erfolgten durch 2 Pneumologen bzw. unter deren Aufsicht durch Weiterbildungsassistenten. Alle Mediastinoskopien wurden von 3 erfahrenen Fachärzten für Thoraxchirurgie durchgeführt, die eine ausgewiesene Expertise für diesen Eingriff besitzen.

Der statistische Vergleich zur diagnostischen Aussagekraft von EBUS-TBNA und Mediastinoskopie erfolgte mit dem Fisher's Exact Test durch die Software Winstat ${ }^{\circledR}$ (www.winstat.de).

\section{Ergebnisse}

Im Zeitraum vom 01.01.2009 bis zum 31.07.2011 wurden 544 konsekutive Lungenkrebspatienten erfasst. Bei diesen erfolgten 111-EBUS-TBNA-Untersuchungen und 88 Mediastinoskopien. In die Analyse wurden alle Untersuchungen einbezogen, die einen pathologischen Tumornachweis erbracht hatten oder in denen ein negativer Befund durch einen chirurgischen Eingriff überprüft wurde. 12 EBUS-TBNA-Untersuchungen und $13 \mathrm{Me}$ -
Tab. 2 Vergleich des diagnostischen Stellenwertes von EBUS und MSK.

\begin{tabular}{|l|lll|}
\hline & EBUS & MSK & $\mathbf{P}$ \\
\hline Diagnostische Genauigkeit & $94 \%$ & $86 \%$ & $\mathrm{P}<0,05$ \\
\hline Neg. präd. Vorhersagewert & $83 \%$ & $83 \%$ & n.s. \\
\hline Sensitivität & $94 \%$ & $58 \%$ & n.s. \\
\hline Prävalenz N2/3 & $84 \%$ & $32 \%$ & $\mathrm{P}<0,05$ \\
\hline Komplikationen & $0 \%$ & $3,4 \%$ & n.s. \\
\hline
\end{tabular}

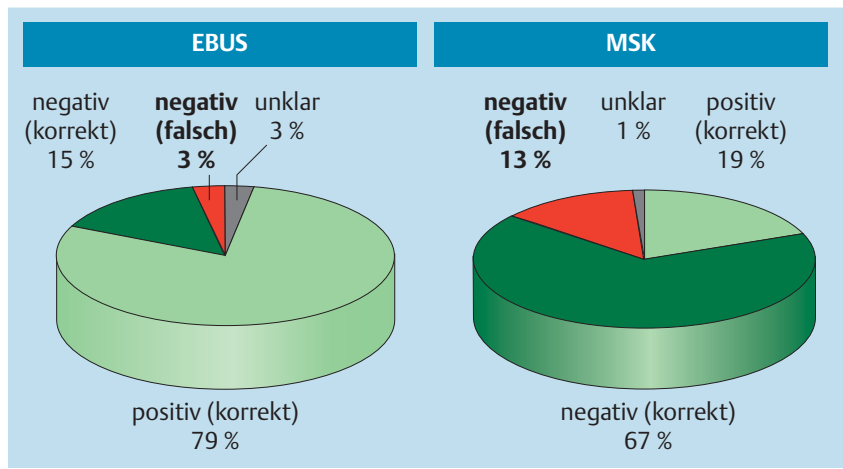

diastinoskopien blieben ohne histologische Kontrolle (meist wegen einer neoadjuvanten Chemotherapie).

Bei drei EBUS-Untersuchungen und einer Mediastinoskopie konnte der Pathologe keine klare Aussage zur Dignität abgeben („tumorsuspekte Zellen“).

Das EBUS-Ergebnis N0/1 war in 15 Fällen korrekt und in 3 falsch negativ (postoperativ N2/3). In 78 Fällen zeigte die EBUS-Untersuchung einen N2/3-Befund. Eine Mediastinoskopie ohne N2/3Nachweis bestätigte sich in der Thorakotomie in 50 Fällen, zehn Fälle waren falsch negativ. In 14 Fällen ergab die Mediastinoskopie ein N2/3-Stadium ( $\bullet$ Tab. 1 ).

Ein Malignitätsnachweis im EBUS bzw. der Mediastinoskopie wurde als korrekt angenommen, daher sind die Spezifität und der positive prädiktive Wert bei beiden Untersuchungsmethoden per definitionem $100 \%$.

Neun Mediastinoskopien erfolgten zum Restaging nach neoadjuvanter Radiochemotherapie. Hierbei war keine Remediastinoskopie. Alle diese Eingriffe ergaben ein korrektes Ergebnis.

Nach einer EBUS-TBNA traten keine relevanten Komplikationen auf. Im Rahmen der Mediastinoskopie ergaben sich 2 Rekurrensparesen und einmal ein postoperatives Vorhofflimmern. Eine statistische Signifikanz in der Komplikationshäufigkeit war nicht gegeben $(p=0,08)$.

Die Prävalenz mediastinaler Lymphknotenmetastasen betrug in der Patientengruppe mit einer EBUS-TBNA 84\%, in der Gruppe mit Mediastinoskopie 33\%.

Die diagnostische Genauigkeit der EBUS-TBNA war mit 93\% signifikant besser als die Genauigkeit der Mediastinoskopie mit 84\% $(\mathrm{p}<0,05)$.

Der negative prädiktive Wert ist beim EBUS und der Mediastinoskopie mit jeweils 93\% identisch ( Tab.2).

Beim EBUS ergaben sich dreimal falsch negative Ergebnisse. In einem Fall lag ein isolierter Befall der paraaortalen Lymphknoten vor, die weder mit EBUS/EUS noch mit einer zervikalen Mediastinoskopie zu erreichen waren. Bei einem weiteren Patienten zeigte sich im OP-Resektat ein isolierter Befall der LK-Station 4R, wobei es sich um einen Single-level-N2 ohne pathologisch vergrößerten Lymphknoten handelte. Nur in einem Fall wurde eine bulky-N2-Erkrankung nicht diagnostiziert. 
Tab.3 Verteilung der histologischen Typen bei mediastinoskopischem bzw. endoskopischem Staging. Angegeben ist die abschließende Histologie am Ende der Primärdiagnostik [15, 16].

\begin{tabular}{|lrc|}
\hline Histologie (ICD-O) & MSK & EBUS \\
\hline Adenokarzinom (8140) & $42 \%$ & $33 \%$ \\
\hline Großzelliges Karzinom (8012) & $7 \%$ & $4 \%$ \\
\hline Plattenepithelkarzinom (8070) & $35 \%$ & $28 \%$ \\
\hline Kleinzelliges Lungenkarzinom (8041) & $8 \%$ & $17 \%$ \\
\hline NSCLC - nicht näher klassifiziert (8020) & $6 \%$ & $13 \%$ \\
\hline Sonstiges Karzinom & $3 \%$ & $5 \%$ \\
\hline
\end{tabular}

Die Zahl der untersuchten Lymphknoten im EBUS lag im Schnitt bei 2,5/Untersuchung (2009: 1,5; 2010: 2,2; 2011: 2,6), bei der MSK bei 7,2/Untersuchung.

Bei der Mediastinoskopie ergaben sich bei 10 Patienten falsch negative Ergebnisse. Ein mediastinaler Tumornachweis fand sich bei der Thorakotomie in den Lymphknotenstationen $4 \mathrm{~L}(2 \times), 4 \mathrm{R}$ $(2 \times), 7(2 \times), 5 / 6(5 \times), 3(3 \times)$.

Die Stadienverteilung im Kollektiv der EBUS-TBNA war folgendermaßen (NSCLC/SCLC): Stadium I+II 23\% (26\%/0\%), Stadium III 50\% (48\%/63\%) und Stadium IV 28\% (25\%/37\%), im Kollektiv der Mediastinoskopie Stadium I+ II 49\% (49\%/50\%), Stadium III $43 \%$ (43\%/50\%) und Stadium IV 8\% (9\%/0\%). Die Stadieneinteilung erfolgte nach der zum Zeitpunkt der Diagnosestellung gültigen UICC-Klassifikation [13,14].

Die histologische Verteilung bei den Untersuchungskollektiven ist in Tab. 3 dargestellt. Herangezogen wurde die abschließende Histologie am Ende der Primärdiagnostik $[15,16]$.

Durch die Tumordokumentation stehen präliminare Daten zum Überleben zur Verfügung. Der Analysezeitpunkt war der 30.08. 2011. Die Patienten mit einem N2/3-Nachweis im EBUS hatten eine mittlere Überlebenszeit von 354 Tagen, mit einem korrekten N2/3-Ausschluss 508 Tage und mit einem falschnegativen N2/3Befund 784 Tage.

\section{Diskussion \\ $\nabla$}

Die EBUS-TBNA, nach Möglichkeit in Kombination mit EUS, ist das Verfahren mit der größten diagnostischen Genauigkeit beim mediastinalen Staging des Lungenkarzinoms. ( Tab.4).

In der retrospektiven Analyse unserer Lungenkarzinompatienten zeigt die EBUS-TBNA eine signifikant höhere diagnostische Genauigkeit als die Mediastinoskopie zur Vorhersage eines N2/3-Status. Die hohe diagnostische Genauigkeit der EBUSTBNA wurde auch in zahlreichen Untersuchungen bestätigt [7, 17-19,20-24]. In einer prospektiven Studie konnte ein Trend zu einer höheren diagnostischen Genauigkeit im N-Staging bei der EBUS-TBNA (89\%) gegenüber einer Mediastinoskopie (79\%) gezeigt werden [25]. In einer weiteren prospektiven Studie zeigte sich eine vergleichbare diagnostische Genauigkeit zwischen endoskopischem Staging und Mediastinoskopie mit jeweils 93\% zur Vorhersage eines mediastinalen Tumorbefalls [26].

Die Komplikationsrate ist beim EBUS in den oben genannten Studien wie auch in unserer Klinik im Trend geringer als bei der MSK. Die bedeutsamste Komplikation der Mediastinoskopie ist die Rekurrensparese [27].

Die diagnostische Genauigkeit der MSK in unserer Untersuchung mit $84 \%$ ist vergleichbar mit anderen aktuellen Studien $[25,26$, 28]
Beim Vergleich zwischen Mediastinoskopie und EBUS-TBNA in dieser Untersuchung muss man einschränken, dass hier Patienten aus zwei unterschiedlichen Kollektiven untersucht wurden. Während die EBUS-TBNA bevorzugt zur Bestätigung eines klinischen N2-Status eingesetzt wurde, erfolgten die meisten Mediastinoskopien bei einem radiologisch unauffälligen Mediastinum. Trotzdem bilden die Daten repräsentativ die Versorgungsqualität an einem nicht universitären Lungenkrebszentrum ab.

In den einschlägigen Leitlinien zum Staging des Lungenkarzinoms kommt jedoch der Mediastinoskopie noch ein zentraler Stellenwert zu. Es ist danach zwar möglich, vor einer Mediastinoskopie eine EBUS-Untersuchung durchzuführen, aber es ist nicht zwingend notwendig $[3,4,28]$.

Die Ergebnisse unserer Arbeit zeigen in Übereinstimmung mit anderen Arbeiten, dass die EBUS-TBNA das erste Verfahren zum histologischen Staging des Mediastinums sein sollte. Bei einer vergleichbaren diagnostischen Genauigkeit weist es eine geringere Komplikationsrate als die Mediastinoskopie auf [25-27]. Ferner kann bei einem initialen N2/3-Nachweis durch eine EBUS-TBNA ein möglicherweise notwendiges Restaging nach einer Induktionstherapie dann durch eine primäre Mediastinoskopie erfolgen. Dieser Algorithmus besitzt die größte Aussagekraft beim mediastinalen Restaging [29]. Wenn initial bereits eine Mediastinoskopie durchgeführt wurde, weist eine erforderliche Remediastinoskopie eine schlechtere diagnostische Aussagekraft auf [30]. Über die Einsatzmöglichkeiten des endoskopischen Ultraschalls zum Restaging gibt es widersprüchliche Studien [31, 32].

Eine methodische Weiterentwicklung stellt die Kombination aus EBUS und EUS dar. Hierdurch ist eine fast vollständige Evaluation des Mediastinum und der hilären Lymphknoten möglich (๑ Abb. 1).

Wir wissen, dass die Prognose des Lungenkarzinoms genauso mit der Anzahl der tumorbefallenen thorakalen Lymphknoten korreliert wie auch mit der Lokalisation [1,33]. In mehreren Studien konnte die diagnostische Überlegenheit einer kombinierten EBUS/EUS-Untersuchung gegenüber dem isolierten Einsatz dieser Methoden gezeigt werden [34-38] ( $\bullet$ Tab.4 und $\bullet$ Tab.5). Dabei war es unerheblich, ob die transoesophageale Untersuchung mit einem EUS-Endoskop [34,35] oder dem EBUS-Bronchoskop $[8,38]$ erfolgte.

Bis jetzt gibt es noch keine Empfehlung über die Anzahl der zu untersuchenden mediastinalen Lymphknoten bei der endoskopischen Diagnostik. Nach den Leitlinien der European Society of Thoracic Surgeons (ESTS) zum Staging des Lungenkarzinoms mittels Mediastinoskopie wird zumindest eine Biopsie der LK-Stationen 4R, 7 und 4L gefordert [3]. Krasnik [39] konnte zeigen, dass dieses Vorgehen auch mit der EBUS-TBNA durchführbar ist. Ein negativer Befund schließt dann mit einer hohen Wahrscheinlichkeit (negativer prädiktiver Wert 95\%) einen mediastinalen Tumorbefall aus. Mit zunehmender Erfahrung im Umgang mit der EBUS-TBNA nimmt die Zahl der untersuchten Lymphknoten zu (๑ Abb. 2).

Ein wichtiges Kriterium für ein definitives diagnostisches Verfahren zum Staging des Lungenkarzinoms ist eine Spezifität von praktisch $100 \%$, da sonst durch falsch positive Befunde Patienten von einer kurativen Operation ausgeschlossen werden. Ein gleichzeitig möglichst hoher negativer prädiktiver Wert bzw. hohe Sensitivität verhindert unnötige Thorakotomien. Aus diesem Grund ist die Kombination von EBUS/EUS das ideale Verfahren zum primären histologischen Staging des Mediastinums. 
Tab. 4 Diagnostischer Stellenwert der Methoden zum mediastinalen Staging. Daten aus Metaanalysen.

\begin{tabular}{|llcclll} 
& Sensitivität & Spezifität & PPV & NPV & Präv & \\
\hline CT & $57 \%$ & $82 \%$ & $56 \%$ & $83 \%$ & $28 \%$ & Tolaza 2003 [43] \\
\hline PET & $84 \%$ & $89 \%$ & $79 \%$ & $93 \%$ & $32 \%$ & Tolaza 2003 [43] \\
\hline MSK & $78 \%$ & $100 \%$ & $100 \%$ & $89 \%$ & $39 \%$ & Detterbeck 2007 [28] \\
\hline EBUS & $90 \%$ & $100 \%$ & $100 \%$ & $80 \%$ & $68 \%$ & Detterbeck 2007 [28] \\
\hline EBUS + EUS & $89 \%$ & $99 \%$ & $99 \%$ & $93 \%$ & $42 \%$ & Tab.5
\end{tabular}

PPV = positiver prädiktiver Wert; NPV= negativer prädiktiver Wert; Präv.= Prävalenz N2/N3.

Tab. 5 Übersicht über Studien zur Kombination EBUS/EUS.

\begin{tabular}{|c|c|c|c|c|c|c|c|c|}
\hline Pat. & LK & Sensitivität & Spezifität & PPV & NPV & Diagn. Genauigkeit & Präv. & \\
\hline 33 & 3,6 & $100 \%$ & $100 \%$ & $100 \%$ & $100 \%$ & $100 \%$ & $61 \%$ & Vilmann 2005 [34] \\
\hline 138 & 2,8 & $93 \%$ & $100 \%$ & $100 \%$ & $97 \%$ & $98 \%$ & $30 \%$ & Wallace 2008 [35] \\
\hline 118 & 3 & $85 \%$ & $100 \%$ & $100 \%$ & $84 \%$ & $92 \%$ & $56 \%$ & Annema 2010 [27] \\
\hline 139 & 4,5 & $95 \%$ & $96 \%$ & $100 \%$ & $95 \%$ & $95 \%$ & $51 \%$ & Herth 2010 [37] \\
\hline 150 & 2,4 & $91 \%$ & $100 \%$ & $100 \%$ & $96 \%$ & $97 \%$ & $26 \%$ & Hwangbo 2010 [38] \\
\hline 120 & 2,6 & $68 \%$ & $98 \%$ & $99 \%$ & $91 \%$ & $91 \%$ & $23 \%$ & Szlubowski 2010 [36] \\
\hline 687 & 3,1 & $89 \%$ & $99 \%$ & $99 \%$ & $93 \%$ & $95 \%$ & $40 \%$ & Summe \\
\hline
\end{tabular}

Pat. = Anzahl Patienten; LK= Anzahl biopsierter Lymphknoten; PPV= positiver prädiktiver Wert; NPV= negativer prädiktiver Wert; Präv. = Prävalenz N2/N3.

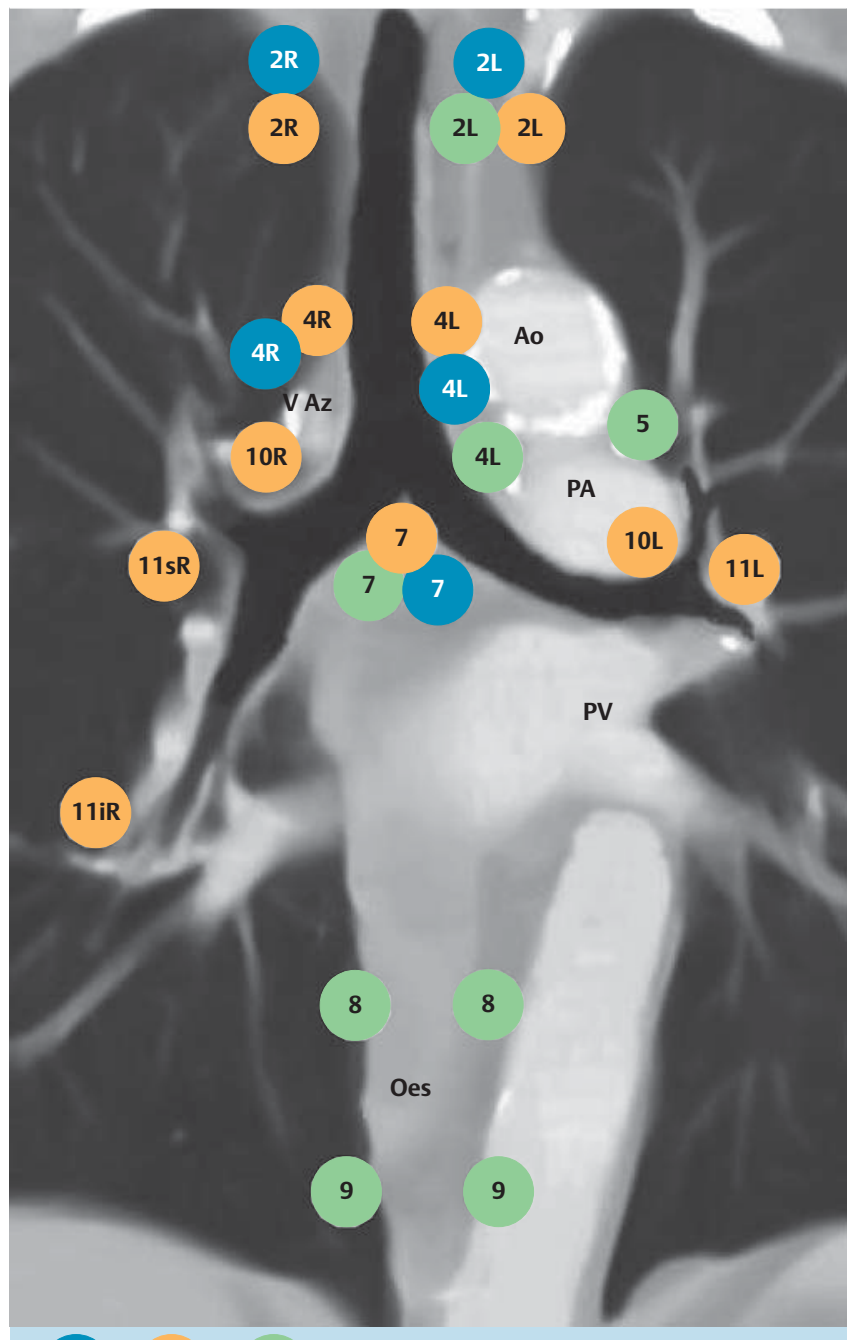

MSK EBUS EUS

Abb. 1 Erreichbare Lymphknotenstationen für Mediastinoskopie, EBUS und EUS. Ao: Aorta; PA: Pulmonalarterie; PV: Pulmonalvene; VAz: Vena Azygos; Oes: Ösophagus.
Nach den derzeitigen Empfehlungen der S3-Leitlinie ist auch nach einem negativen EBUS eine Mediastinoskopie erforderlich. Die Kombination aus EBUS/EUS und ggf. nachfolgender Mediastinoskopie steigert die Genauigkeit des mediastinalen Stagings gegenüber einer alleinigen Mediastinoskopie. Bei einem negativen EBUS/EUS sind hierbei aber 10 Mediastinoskopien erforderlich, um einen zusätzlichen N2-Befall nachzuweisen [27]. Da die Mediastinoskopie aber eine klinisch relevante Komplikationsrate aufweist, muss das Risiko dieses Eingriffs kritisch gegenüber dem Risiko einer unnötigen Thorakotomie infolge eines übersehenen prognostisch inoperablen fortgeschrittenen N2-Befalls bei Verzicht auf eine MSK abgewogen werden.

Bei einem in der Bildgebung unauffälligen Mediastinum mit einer geringen Prävalenz eines N2-Stadiums kann nur selten durch eine die EBUS-TBNA ergänzende Mediastinoskopie ein mediastinaler Tumornachweis gelingen. Dies bestätigte Tournoy in einer Subgruppenanalyse der ASTER-Studie [40], aber auch Herth [41].

Da der prognostische Nutzen einer Tumorresektion bei einer hilären und insbesondere mediastinalen Tumorausbreitung abnimmt, ist bei einem hohen OP-Risiko ein besonders sorgfältiges Staging erforderlich. Auch hier ist in der Regel eine Mediastinoskopie bei einem negativen EUS/EBUS indiziert.

In der Diskussion zwischen EBUS und Mediastinoskopie wird immer wieder darauf hingewiesen, dass die MSK einen höheren negativen prädiktiven Wert hat. Dieser ist aber auch entscheidend von der Prävalenz des mediastinalen Lymphknotenbefalls abhängig. Vor allem die frühen Studien zur EBUS-TBNA sind bei Patienten mit einem vermuteten N2-Befall erfolgt, sodass trotz einer hohen diagnostischen Genauigkeit ein unbefriedigender negativer prädiktiver Wert resultierte [20]. Die in unseren Untersuchungen erhobenen präliminaren Daten zur Überlebenszeit bei Patienten mit gesichertem N2-Befall und einem falsch negativen EBUS-Befund zeigen, dass diese Patienten eine deutlich bessere Prognose haben als die Patienten mit einem positiven EBUSBefund. Für eine statistische Analyse sind hier die Fallzahlen aber zu gering. Der hier zu beobachtende Trend findet sich aber auch in einer größeren Analyse von Sakari bestätigt [42]. In einer retrospektiven Analyse von Patienten mit einem negativen EBUSBefund, aber nachgewiesenem N2-Befall in der Thorakotomie re- 


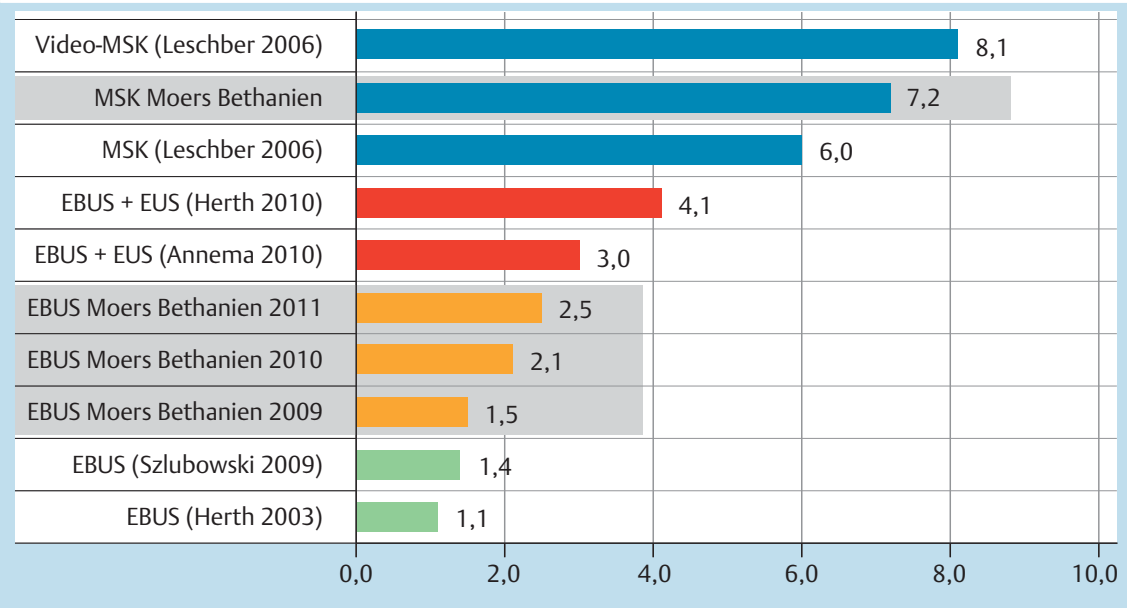

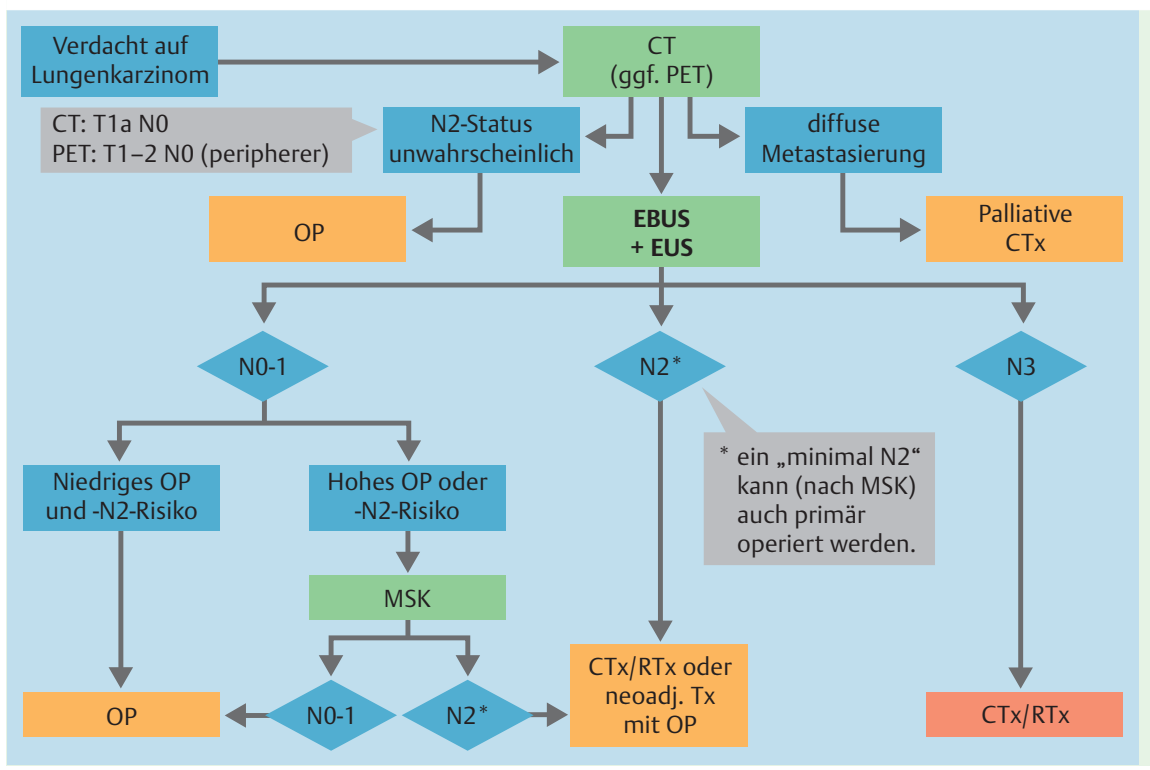

Abb. 2 Anzahl untersuchter Lymphknoten in ausgewählten Arbeiten zum mediastinalen Staging im Vergleich zu den Ergebnissen im Lungenzentrum Bethanien Moers. sultierte eine 5-Jahres-ÜLR von 77\% bei einem CT-morphologisch unauffälligen Mediastinum und von $54 \%$ bei vergrößerten Lymphknoten. Diese überaus guten Überlebensraten für Patienten im Stadium III zeigen, dass die EBUS-TBNA ein exzellentes Tool für das mediastinale Staging und zur Selektion von Patienten für eine operative Therapie darstellt.

\section{Fazit}

Die EBUS-TNA besitzt eine überlegene Genauigkeit für das mediastinale Staging. Wenn durch die Bildgebung ein N2-Status nicht ausgeschlossen ist, sollte die Kombination aus EBUS und EUS die Methode der 1. Wahl zur histologischen Untersuchung des Mediastinums sein. Bei einem negativen Ergebnis kann bei einer geringen Wahrscheinlichkeit für einen N2-Befall und einem geringen OP-Risiko auf eine Mediastinoskopie verzichtet werden. In unserer Klinik besitzt daher der EBUS (in Kombination mit dem EUS) einen zentralen Stellenwert

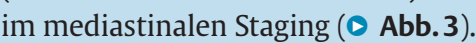

\section{Interessenkonflikt}

Die Autoren geben an, dass kein Interessenkonflikt vorliegt.

\section{Literatur}

1 Rush V, Crowley J, Giroux D et al. The IASLC lung cancer staging project: Proposals for the revision of the $\mathrm{N}$ descriptors in the forthcoming seventh edition of the TNM classification for lung cancer. J Thorac Oncol 2007; 2: 603-612

2 Silvestri GA, Gould MK, Margolis ML et al. Noninvasive staging of nonsmall cell lung cancer: ACCP evidenced-based clinical practice guidelines (2nd edition). Chest 2007; 132: S178-S201

3 De Leyn $P$, Lardinois $P$, Van Schill $P$ et al. ESTS guidelines for preoperative lymph node staging for non-small cell lung cancer. Eur J Cardiothorac Surg 2007; 32: $1-8$

4 Goeckenjan G, Sitter $H$, Thomas $M$ et al. Prävention, Diagnostik, Therapie und Nachsorge des Lungenkarzinoms. Interdisziplinäre S3-Leitlinie der Deutschen Gesellschaft für Pneumologie und Beatmungsmedizin und der Deutschen Krebsgesellschaft. Pneumologie 2010; 64 (Suppl. 02): $1-164$

5 Hürter Th, Hanrath P. Endobronchiale Sonographie zur Diagnostik pulmonaler und mediastinaler Tumoren. Dtsch med Wochenschr 1990; 115: $1899-1905$

6 Herth F, Becker H, Ernst A. Ultrasound-guided transbronchial needle aspiration. Chest 2003; 123: 604-607

7 Groth S, Andrade R. Endobronchial and Endoscopic Ultrasound-Guided Fine-Needle Aspiration: A Must for Thoracic Surgeons. Ann Thorac Surg 2010; 89: 2079-2083 
8 Herth F, Krasnik M, Kahn N et al. Combined endoscopic-endobronchial ultrasound-guided fine-needle aspiration of mediastinal lymph nodes through a single bronchoscope in 150 patients with suspected lung cancer. Chest 2010; 138: 790-794

9 Wallace M, Pascual J, Raimondo $M$ et al. Minimal invasive endoscopic staging of suspected lung cancer. JAMA 2008; 299: 540-546

10 Tournoy K, Annema J, Krasnik $M$ et al. Endoscopic and endobronchial ultrasonography according to the proposed lymph node map definition in the seventh edition of the tumor, node, metastasis classification for lung cancer. J Thorac Oncol 2009; 4: 1576-1584

11 Mountain C, Dresler C. Regional lymph node classification for lung cancer staging. Chest 1997; 111: 1718-1723

12 Goldstraw P. IASLC Staging Manual in Thoracic Oncology. Editorial Rx Press; 2009: 978-0-9799274-3-0

13 UICC TNM Classification of Malignant Tumours. 6th edition. WileyBlackwell; 2002

14 UICC TNM Classification of Malignant Tumours. 7th edition. WileyBlackwell; 2009

15 Travis WD, Brambilla E, Muller-Hermelink HK, Harris CC Hrsg. World Health Organisation Classification of Tumours. Pathology and Genetics of the Tumours of the Lung, Pleura, Thymus and Heart Lyon: IARC Press; 2004: 9-124

16 Travis W, Brambilla E, Noguchi M et al. International Association for the Study of Lung Cancer/American Thoracic Society/European Respiratory Society international multidisciplinary classification of lung adenocarcinoma. J Thorac Oncol 2011; 6: 244-285

17 Varela-Lema L, Fernández-Villar A, Ruano-Ravina A. Effectiveness and safety of endobronchiale ultrasound-transbronchial needle aspiration: a systematic review. Eur Respir J 2009; 33: 1156 - 1164

18 Darwiche K, Wagner M, Theegarten D. Aktueller Stellenwert des endobronchialen Ultraschalls (EBUS). Pneumologie 2011; 65: 537-548

19 Yasufuku K, Chiyo M, Koh E et al. Endobronchial ultrasound guided transbronchial needle aspiration for staging of lung cancer. Lung Cancer 2005; 50: 347 - 354

20 Herth FJ, Eberhardt R, Vilmann $P$ et al. Real-time endobronchial ultrasound guided transbronchial needle aspiration for sampling mediastinal lymph nodes. Thorax 2006; 61: 795-798

21 Vincent BD, El-Bayoumi E, Hoffman B et al. Real-time endobronchial ultrasound-guided transbronchial lymph node aspiration. Ann Thorac Surg 2008; 85: 224-230

22 Groth SS, Whitson BA, D'Cunha J et al. Endobronchial ultrasound-guided fineneedle aspiration of mediastinal lymph nodes: a single institution's early learning curve. Ann Thorac Surg 2008; 86: 1104-1110

23 Gilbert S, Wilson DO, Christie NA et al. Endobronchial ultrasound as a diagnostic tool in patients with mediastinal lymphadenopathy. Ann Thorac Surg 2009; 88: 896-902

24 Omark Petersen H, Eckardt J, Hakami A et al. The value of mediastinal staging with endobronchial ultrasound-guided transbronchial needle aspiration in patients with lung cancer. Eur J Cardiothorac Surg 2009; 36: $465-468$

25 Ernst A, Anatham D, Eberhardt $R$ et al. Diagnosis of mediastinal adenopathy - real-time endobronchial ultrasound guided needle aspiration versus mediastinoscopy. J Thorac Oncol 2008; 3: 577 -582

26 Yasufuku K, Pierre A, Darling G et al. A prospective controlled trial of endobronchial ultrasound-guided transbronchial needle aspiration compared with mediastinoscopy for mediastinal lymph node staging of lung cancer. J Thorac Cardiovasc Surg 2011; 142: 1393 -1400
27 Annema J, van Meerbeeck J, Rintoul R. Mediastinoscopy vs endosonography for mediastinal nodal staging of lung cancer. JAMA 2010; 304: $2245-2252$

28 Detterbeck $F C$, Jantz $M A$, Wallace $M$ et al. Invasive mediastinal staging of lung cancer: ACCP evidence-based clinical practice guidelines (2nd edition). Chest 2007; 132: 202S-220S

29 de Cabanyes Candela S, Detterbeck F. A systematic review of restaging after induction therapy for stage IIIa lung cancer. Prediction of pathologic stage. J Thorac Oncol 2010; 5: 389-398

30 Stamatis G, Fechner S, Hillejan L. Die Re-Mediastinoskopie als diagnostisches Verfahren zur Restadiierung des Bronchialkarzinoms. Pneumologie 2005; 59: 862 - 866

31 Herth F, Annema J, Eberhardt $R$ et al. Endobronchial Ultrasound With Transbronchial Needle Aspiration for Restaging the Mediastinum in Lung Cancer. J Clin Oncol 2008; 26: 3346-3350

32 Szlubowski A, Herth F, Soja J. Endobronchial ultrasound-guided needle aspiration in non-small-cell lung cancer restaging verified by the transcervical bilateral extended mediastinal lymphadenectomy - a prospective study. Eur J Cardiothorac Surg 2010; 37: 1180-1184

33 Lee J, Lee C, Park I et al. Number of metastatic lymph nodes in resected non-small cell lung cancer predicts patient survival. Ann Thorac Surg 2008; 85: 211-215

34 Vilmann P, Krasnik M, Larsen S et al. Transoesophageal endoscopic ultrasound-guided fine-needle aspiration (EUS-FNA) and endobronchial ultrasound-guided transbronchial needle aspiration (EBUS-TBNA) Biopsy: a combined approach in the evaluation of mediastinal lesions. Endoscopy 2005; 37: 833-839

35 Wallace $M$, Pascual J, Raimondo $M$ et al. Minimally invasive endoscopic staging of suspected lung cancer. JAMA 2008; 299: 540-546

36 Szlubowski A, Zielinski M, Soja J et al. A combined approach of endobronchial and endoscopic ultrasound-guided needle aspiration in the radiologically normal mediastinum in non-small-cell lung cancer staging a prospective trial. Eur J Cardiothorac Surg 2010; 37: 1175 - 1179

37 Herth F, Krasnik M, Kahn N et al. Combined endoscopic-endobronchial ultrasound-guided fine-needle aspiration of mediastinal lymph nodes through a single bronchoscope in 150 patients with suspected lung cancer. Chest 2010; 138: 790 - 794

38 Hwangbo B, Lee G, Lee HS et al. Transbronchial and Transesophageal Fine-Needle Aspiration Using an Ultrasound Bronchoscope in Mediastinal Staging of Potentially Operable Lung Cancer. Chest 2010; 138 795-802

39 Krasnik M, Skov B, Mellemgaard A et al. Is mediastinoscopy necessary in patients with negative EBUS-TBNA - a challenge to the ESTS-guidelines for staging lung cancer. WCLC 2011 034.02. J Thorac Oncol 2011; 6: 425

40 Tournoy K, Rintoul R, Dooms C et al. Can mediastinoskopy after negative endosonography in lung cancer be emitted? Subanalysis of ASTER with focus on CT ERS-Kongress: 2011: P1972

41 Herth F, Ernst A, Eberhardt $R$ et al. Endobronchial ultrasound-guided transbronchial needle aspiration of lymph nodes in the radiololgically normal mediastinum. Eur Respir J 2006; 28: 910-914

42 Sakairi $Y$, Hoshino H, Nakajima $T$ et al. Clinical outcome of $\mathrm{cNO}$ nonsmall cell lung cancer patients staged by thinslice CT, FDG_PET, and EBUS-TBNA. J Clin Oncol 2011; 29: abstr 7052

43 Toloza E, Harpole L, McCrory D. Noninvasive Staging of Non-small Cell Lung Cancer - A Review of the Current Evidence. Chest 2003; 123: $137-146$ 